\title{
Interstitial Chromosome 3p14.1 Deletion due to a Maternal Insertion: Phenotype and Association with Balanced Parental Rearrangement
}

\author{
Catherine Hajek $^{\mathrm{a}}$ Jia-Chi Wang ${ }^{c} \quad$ Loretta W. Mahon $^{c} \quad$ Ariadna Martinez ${ }^{\mathrm{a}}$ \\ Sulagna C. Saitta ${ }^{a, b}$ \\ ${ }^{a}$ Division of Medical Genetics, Department of Pediatrics, Cedars-Sinai Medical Center and ${ }^{\mathrm{b}}$ Department of \\ Pediatrics, David Geffen School of Medicine at UCLA, Los Angeles, Calif., and ${ }^{\complement}$ Cytogenetics Laboratory, Quest \\ Diagnostics Nichols Institute, San Juan Capistrano, Calif., USA
}

\section{Established Facts}

- There is a described phenotype for interstitial deletion at 3 p14.1 which our case supports.

\section{Novel Insights}

- Our case is the only one reported in which the proband's deletion derives from a maternal insertion a finding not apparent by chromosomal microarray and only diagnosable by FISH analysis.

- The use of molecular cytogenetic studies for structural analysis and parental studies is necessary to elucidate the underlying cause of chromosomal abnormalities and define the recurrence risk for families.

\section{Key Words}

Autosomal deletion - Blepharophimosis · Developmental delay · Interstitial 3p deletion - Maternal insertion .

Microphthalmia

\begin{abstract}
Interstitial deletions of 3p14p12 are rare chromosome abnormalities. We present a patient with multiple congenital anomalies and a 15.4-Mb interstitial loss of chromosome 3p14p12 detected by chromosomal microarray (CMA). Our patient shared many phenotypic features with other reported cases involving the same region including prominent forehead, short palpebral fissures, hand and foot anomalies,
\end{abstract}

genital abnormalities, and bilateral hearing loss. Given the clinical similarity of these cases with significant overlap of the deleted regions, it is likely that the phenotype is related to the deletion of specific genes within the region. Further molecular cytogenetic investigation revealed that our patient's rearrangement was derived from a cryptic insertion of a segment of chromosome $3 p$ into chromosome $18 q$ in the mother, which was balanced and therefore not visible on the mother's CMA. To our knowledge, this finding has not been previously reported. This case illustrates the importance of using molecular cytogenetics for structural analysis and parental studies. CMA is commonly the first-line study in patients with multiple congenital anomalies; however, it is not the appropriate modality to define a structural rearrange-

\section{KARGER}

E-Mail karger@karger.com

www.karger.com/msy (c) 2016 S. Karger AG, Basel

1661-8769/16/0071-0043\$39.50/0
Sulagna Saitta, MD, PhD

Keck School of Medicine at USC 2100 West 3rd Street

Los Angeles, CA 90057 (USA)

E-Mail ssaitta@chla.usc.edu 
ment that may be the cause of a deletion. The use of adjunct studies to define the mechanism of an identified copy number aberration has direct clinical application: to identify the underlying cause of the chromosomal abnormality and to define the recurrence risk. Additionally, this case adds to the current body of work regarding a recurrent phenotype that can be attributed to interstitial chromosome $3 p$ deletions, which may help define the phenotypic implications of deletions in this region and support early clinical management.

(C) 2016 S. Karger AG, Basel

Interstitial deletions of $3 \mathrm{p} 14 \mathrm{p} 12$ are rare chromosome abnormalities, with less than 30 cases reported in the literature [Dimitrov et al., 2015]. Here, we present a patient with multiple congenital anomalies and a $15.4-\mathrm{Mb}$ interstitial loss of chromosome $3 \mathrm{p} 14 \mathrm{p} 12$ detected by chromosomal microarray (CMA). Further molecular cytogenetic investigation revealed that the patient's rearrangement was derived from a cryptic insertion of a segment of chromosome $3 p$ into chromosome $18 \mathrm{q}$ in the mother, which was balanced and therefore not visible on the mother's CMA. This finding, which to our knowledge has not been previously reported, underscores the continued utility of structural cytogenetic analysis for defining the mechanism of rearrangement and for providing an accurate recurrence risk.

\section{Clinical Report}

The patient was a full-term male infant, born to a 35-year-old G1P0 woman with a history of infertility. This pregnancy was the result of a second in vitro fertilization attempt and was originally a dichorionic/diamniotic twin pregnancy with the demise of one twin at 9 weeks of gestation. The mother received routine prenatal care at an outside institution, and the fetus was found to have multiple congenital anomalies on ultrasound including intrauterine growth retardation, vermian agenesis with an enlarged 3rd ventricle, microphthalmia, asymmetric orbits, clenched fingers, dilated loops of bowel, possible clubbed feet, and possible hypospadias. The mother declined amniocentesis, and noninvasive prenatal testing was deemed inappropriate because of the original twin pregnancy. Labor was induced at $381 / 7$ weeks for intrauterine growth retardation, and the infant was delivered by caesarean section after arrest of dilatation and nonreassuring fetal heart tones. There was no family history of birth defects, mental retardation, hearing problems, blindness, or cardiac anomalies, and the couple is nonconsanguineous.

At birth, Apgar scores were 8 at 1 and also at $5 \mathrm{~min}$. The initial examination was notable for poor neurologic tone. The infant's growth parameters showed a length of $55.5 \mathrm{~cm}$ (99th percentile), $2,744 \mathrm{~kg}$ (9th percentile) and a head circumference of $33 \mathrm{~cm}$ (12.5th percentile).
Further investigation noted a wide, diamond-shaped anterior fontanelle that was splayed into the temporal areas bilaterally. There was hair on the forehead, including 2 formal hair whorls bilaterally, with a prominent, bossed forehead. The nasal bridge appeared pinched. The eyelids were small with shortened palpebral fissures and blepharophimosis. The left eye was recessed into the orbit, consistent with microphthalmia. The left eyelid appeared to be fused. The mouth was downturned. The palate had a very high arch, and there was micrognathia. The ears were normally positioned, though the right ear showed a poorly formed hypoplastic helix.

The chest examination showed wide-spaced nipples and a 2/6 holosystolic murmur. The abdominal examination was unremarkable, with no abdominal wall defects. The spine was straight with a sacral hair tuft. On genitourinary examination, there was a microphallus with prominent scrotal ridge and bilateral cryptorchidism. The upper extremities were proportional in size. The thumbs were hypoplastic bilaterally. There were club feet bilaterally.

The neurological examination revealed significant central hypotonia. The upper and lower extremities were flexed with normal movement. The hands were clenched and contracted. The toes were clenched similarly to the hands. The infant's suck was poor.

An echocardiogram revealed bidirectional shunting at a patent foramen ovale, with a redundant atrial septum. There was unobstructed forward flow through the right ventricular outflow tract into normally sized branch pulmonary arteries.

Brain MRI demonstrated a phthisis bulbi appearance of the globe of the left eye. The cerebellar vermis was noted to be slightly hypoplastic. The cerebellar hemispheres themselves were well formed. The infant failed his newborn hearing screen. On followup examination, the infant was noted to have significant motor and developmental delay and persistent hypotonia.

\section{Materials and Methods}

Peripheral blood was obtained from the patient and his mother. Cytogenetic analysis was initially performed on the proband by a different laboratory and was reported as normal using standard G-banding. CMA studies on both the infant and his mother were performed using an oligo-SNP array (Affymetrix CytoScan HD) that included 2.67 million probes, with 1.9 million copy number probes and 750,000 SNP probes with an overall average interprobe distance of 1,150 base pairs. Thresholds for genome-wide screening were set at $>200 \mathrm{~kb}$ for gains, $>50 \mathrm{~kb}$ for losses, and areas $>5$ $\mathrm{Mb}$ in size for region of homozygosity. The FISH assay described was performed with a BAC probe (BlueGnome/Illumina, San Diego, Calif., USA) specific for chromosome 3p14.1 (RP11-413H16; chr3: 64,744,592-64,933,582; hg19) in accordance with the manufacturer's instructions.

\section{Results}

CMA identified an interstitial deletion in the short arm of chromosome 3 for the proband. The deletion was $15.4 \mathrm{Mb}$ in size with genomic coordinates at chr3: 


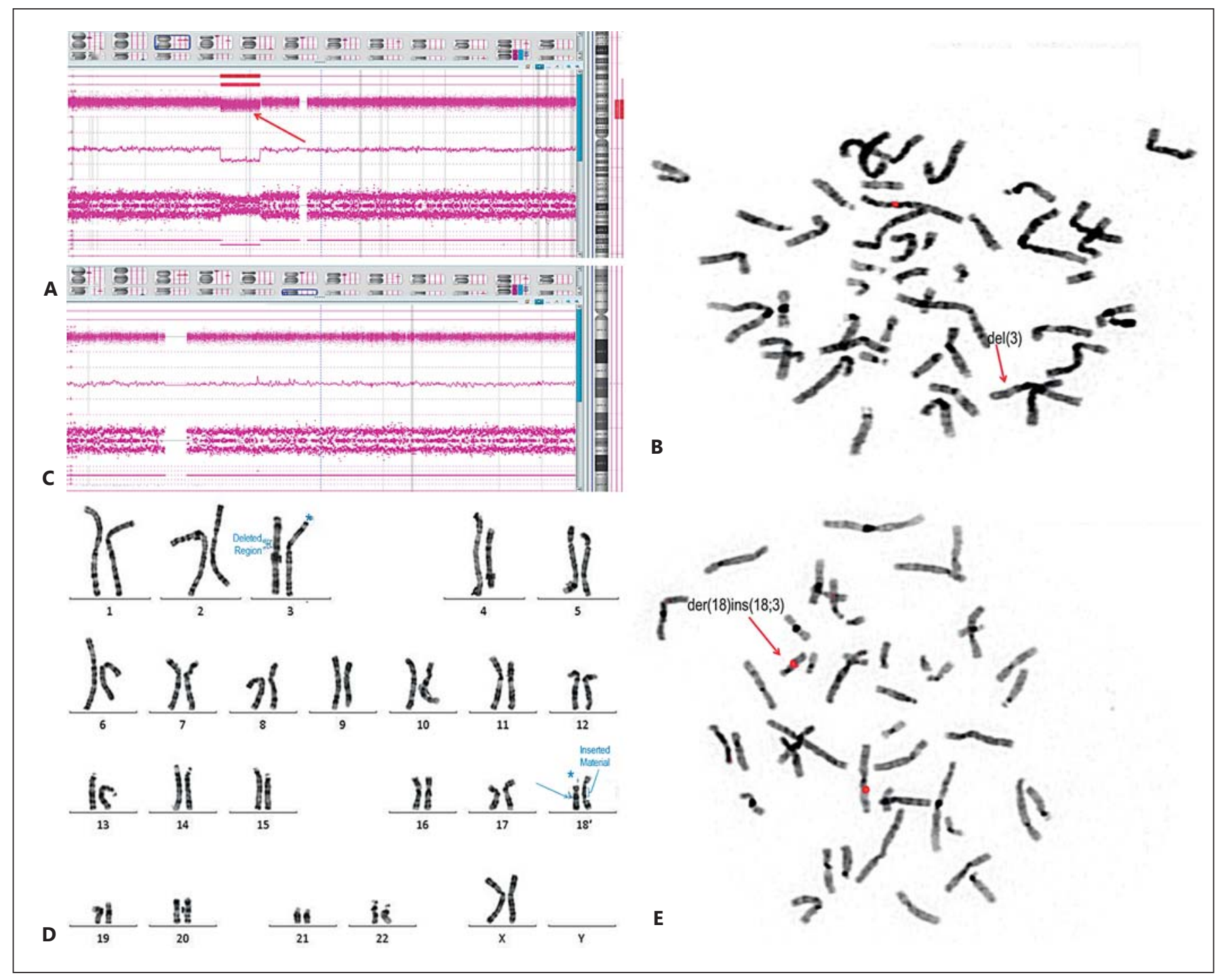

Fig. 1. A CMA of the proband showing an interstitial heterozygous deletion at 3p14.2p12.3 (chr3: 59,854,516-75,236,053; hg19) indicated by the arrow. B FISH analysis using BAC probe RP11413H16 at 3p14.1 (chr3: 64,744,592-64,933,582; hg19) labeled in red revealed only one signal consistent with a deletion at one of the 3 p14.1 regions (arrow). C CMA of the proband showing a normal result on chromosome 18 with no copy number variation. D Ma- ternal karyotype: 46,XX,ins(18;3)(q12.2;p12.3p14.2). The asterisk indicates the normal chromosomes 3 and 18. The arrows indicate the region where the insertion occurred. Deleted and inserted materials are marked with square brackets. E Maternal FISH analysis using the RP11-413H16 probe confirming an insertional translocation from $3 p$ to $18 \mathrm{q}$ (arrow).
59,854,516-75,236,053 [hg19] (fig. 1A). This finding was confirmed by FISH analysis using a labeled BAC probe located within the deleted interval, which showed an absent signal in one of the chromosome 3p14.1 regions (fig. 1B), in all examined metaphase cells. Chromosome 18 was normal by CMA (fig. 1C). The maternal karyotype showed an insertion of a segment from the short arm of chromosome 3, 3p12.3p14.2, into the long arm of one copy of chromosome 18 at 18q12.2 (fig. 1D), with a nomenclature of 46,XX,ins(18;3)(q12.2;p12.3p14.2). BACFISH analysis of the mother's peripheral blood chromosomes using the same BAC probe employed for the patient's FISH study above confirmed an insertion of this probe into 18q12.2 (fig. 1E). A paternal sample was not available; however, the proband's rearrangement was explained by the maternal findings. 


\section{Discussion}

There have been a few recent cases of interstitial deletions of the short arm of chromosome 3 reported in the literature [Țuțulan-Cunită et al., 2012; Schwaibold et al., 2013; Dimitrov et al., 2015]; however, to the best of our knowledge, there have been no prior reports of an interstitial deletion derived from a balanced parental chromosomal insertion. The few previously reported cases of deletion at 3p14 do share similar phenotypes to our patient, including a broad forehead, hypertelorism, down-slanting palpebral fissures, cardiac defect, contractures, hearing loss, and genitourinary abnormalities [Dimitrov et al., 2015].

A series of 14 patients with proximal interstitial $3 p$ deletions and overlapping phenotypic features was described; however, phenotypes varied depending on the size of the deletion and the genes involved [Dimitrov et al., 2015]. Of these, 3 cases had similar genomic locations and large size of deletion. One patient had a de novo $3 \mathrm{p} 14.2 \mathrm{p} 12.3$ deletion of $16 \mathrm{Mb}$, another had a 3p14.2p12.1 deletion of $27.2 \mathrm{Mb}$, and the third had a 3p14.2p12.3 deletion of 15.4 Mb. Țuțulan-Cunită et al. [2012] also reported a $12.5-\mathrm{Mb}$ deletion in the short arm of chromosome 3 involving the region $3 \mathrm{p} 14.1 \mathrm{p} 12.3$. These 4 patients all shared many phenotypic features with our proband, including a prominent forehead, short palpebral fissures, hand and foot anomalies, genital abnormalities, and bilateral hearing loss (table 1). The table summarizes the physical findings of the 5 patients with comparable deletion sizes and regions. Given the clinical similarity of these cases with significant overlap of the deleted regions, it is likely that the phenotype is related to the deletion of specific genes within the region.

The most common phenotypic features described in interstitial $3 p$ deletions are intellectual disability (ID), developmental delay (DD), and autism. These features are suggested to be related to haploinsufficiency of the FOXP1 gene (OMIM 613670) at 3p13. FOXP1 has been associated with several reports of deletions involving this region in patients with ID and DD. Le Fevre et al. [2013] reported a single patient and literature review of several others with FOXP1 deletions and severe ID/DD as well as severe speech delay. Their case series identified that expressive speech is severely affected. The developmental outcome in our patient based on investigation was expected to include significant delays. It is not clear how our patient's speech will develop, but knowledge of this association will help guide speech and language therapy. Another important feature described in interstitial deletions of $3 p$ is sensorineural hearing loss, which is thought to be related to haploinsufficiency of the MITF gene (OMIM 156845) at $3 \mathrm{p} 14 \mathrm{p} 13$. This gene is considered causal for Waardenburg syndrome type 2A hearing loss [Nobukuni et al., 1996]. Both FOXP1 and MITF are genes likely to exhibit haploinsufficiency based on their OMIM haploinsufficiency scores of 0.13 and 0.97 , respectively. Involvement of MITF further underscores the need for careful and sequential developmental follow-up and intervention for the patient. The phenotype for proximal 3p14p12 interstitial deletions is evolving as additional cases are reported. However, most cases reported in the literature do not share the severity of phenotype seen in our patient.

One of the most striking physical features of our patient is blepharophimosis. This feature is often associated with deletion of the FOXL2 gene at 3p23, but this gene was not within the deleted region in our patient. FOXL2 belongs to the same family as FOXP1 (which is deleted in our patient) and has a role in PITX 3 transcription. Mutations in PITX3 have been associated with ophthalmologic malformations, most commonly congenital cataracts. Since no patients with FOXP1 mutations have developed significant eye abnormalities [Le Fevre et al., 2013], further studies are required to demonstrate whether FOXP1 is responsible for the eye phenotype in our patient. It will be important to determine the contribution of several other neurodevelopmental genes that are included in the deletion, including CADPS, CNTN3, SYNPR, FEZF2, PDZRN3, and ATXN7 to the phenotype.

The frequency of insertional translocations has been estimated at $\sim 1: 80,000$ patients based on the karyotype alone [Van Hemel and Eussen, 2000]. However, the study published by Kang et al. [2010], in which 18,000 cases were evaluated, revealed that the frequency of such insertions was $\sim 1: 450$ by using array-CGH technology. These cases were further categorized as (1) simple intrachromosomal and interchromosomal insertional translocations resulting in pure segmental trisomy, (2) complex insertional translocations involving more than one abnormality, and (3) deletions inherited from a parent with a balanced rearrangement that resulted in pure segmental monosomy. Of the 40 cases identified, only 4 fell into the third category and none involved chromosome 3 . Here, we demonstrated the utility of FISH analysis in the discovery of a cryptic parental insertion involving 2 chromosomes. In the era of CMA, FISH analysis is a technique that may be overlooked; nonetheless, it detected the parental origin of our patient's chromosomal deletion and determined its underlying mechanism. Thus, the frequency of balanced insertional rearrangements may be underestimated due to the lack of availability and exami- 
Table 1. Features present in large $3 p$ deletions

\begin{tabular}{|c|c|c|c|c|c|}
\hline & \multirow[t]{2}{*}{ Our patient } & \multicolumn{3}{|c|}{ Dimitrov et al., 2015} & \multirow{2}{*}{$\begin{array}{l}\text { Țuțulan-Cunită } \\
\text { et al., } 2012\end{array}$} \\
\hline & & case 1 & case 2 & case 3 & \\
\hline Microarray coordinates & $\begin{array}{l}59,854,516- \\
75,236,053\end{array}$ & $\begin{array}{l}58,573,617- \\
74,591,661\end{array}$ & $\begin{array}{l}59,679,032- \\
86,907,648\end{array}$ & $\begin{array}{l}62,021,974- \\
77,413,410\end{array}$ & $\begin{array}{l}65,317,864- \\
77,902,945\end{array}$ \\
\hline Size & $15.4 \mathrm{Mb}$ & $16 \mathrm{Mb}$ & $27.2 \mathrm{Mb}$ & $15.4 \mathrm{Mb}$ & $12.5 \mathrm{Mb}$ \\
\hline Severe developmental delay & + & + & + & + & + \\
\hline Wide anterior fontanelle & + & & & + & \\
\hline High broad forehead & + & + & + & & + \\
\hline Midfacial hypoplasia & & + & & & \\
\hline Short nose & & + & + & & \\
\hline Microphthalmia & + & & + & & \\
\hline Micrognathia & + & & + & & \\
\hline Low-set ears & & + & + & & \\
\hline Small mouth & & + & & & \\
\hline Enlarged ventricles & & + & + & + & \\
\hline Loss of white matter volume & & + & + & & \\
\hline Increased lower extremity muscle tone & + & + & + & + & \\
\hline Clenched hands or feet & + & & & & \\
\hline Contractures & + & + & + & + & \\
\hline Cryptorchidism & + & & + & & + \\
\hline Microphallus & + & & + & & + \\
\hline Scrotal or labial abnormalities & + & & + & + & + \\
\hline
\end{tabular}

$\mathrm{ASD}=$ Atrium septal defect; AVSD = atrio-ventricular septum defect; PDA = patent ductus arteriosus; $\mathrm{PFO}=$ patent foramen ovale; $+=$ present.

nation of parental samples utilizing a technique to assess chromosomal structure.

Our case underscores the continued need to perform structural molecular cytogenetic analysis to define the mechanism of deletions and duplications in order to more accurately estimate the risk to future pregnancies. Considering a 1:1:1:1 prediction of meiotic segregants, the risk of having an unbalanced complement (duplication or deletion) in future pregnancies would be 50\% [Youings et al., 2004]. Given this significant risk for all future pregnancies, prenatal diagnosis should be offered to carrier couples for all future pregnancies. Array CGH performed with chorionic villus sampling or amniocentesis should be able to identify submicroscopic unbalanced deletions or duplications in an affected fetus [Hanemaaijer et al., 2012]. In addition, karyotype and specific FISH analysis should be offered for other family members, particularly maternal siblings of child-bearing age in our case. It is possible that the history of infertility prompting in vitro fertilization in our patient's mother is related to her structural chromosomal rearrangement, and pre-implantation genetic diagnosis should also be considered to screen for rearrangements. 


\section{Conclusion}

An interstitial 3p14p12 deletion is a rare finding, particularly when resulting from a cryptic parental insertion. This case illustrates the continuing importance of using molecular cytogenetics for structural analysis and of parental studies. Here, targeted FISH analysis in the mother was the only available methodology to identify the underlying cause of the chromosomal abnormality and to define the recurrence risk for this family. While CMA is the accepted and best first-line study in patients with multiple congenital anomalies [Miller, 2010], it is not the appropriate modality to define a structural rearrangement that may be the cause of a deletion. The use of adjunct studies to define the mechanism of an identified copy number aberration has direct clinical application. Additionally, this case adds to the current body of work regarding a recurrent phenotype that can be attributed to interstitial chromosome $3 \mathrm{p}$ deletions, which may help understand the phenotypic implications of deletions in this region and support early clinical management.

\section{Acknowledgments}

We thank Jeff Radcliffe, Dr. Jianling Ji and Dr. Jaclyn Biegel for critical reading of the manuscript. C.H. is a trainee of the UCLA Intercampus Medical Genetics Training Program, Los Angeles, Calif., USA.

\section{Statement of Ethics}

Appropriate approval has been obtained from the first author's institution. Informed consent was obtained from the patient's mother.

\section{Disclosure Statement}

The authors have no conflicts of interest to disclose.

\section{References}

Dimitrov BI, Ogilvie C, Wieczorek D, Wakeling E, Sikkema-Raddatz B, et al: 3p14 deletion is a rare contiguous gene syndrome: report of 2 new patients and an overview of 14 patients. Am J Med Genet A 167:1223-1230 (2015).

- Hanemaaijer N, Sikkema-Raddatz B, van der Vries G, Dijkhuizen T, Hordijk R, et al: Practical guidelines for interpreting copy number gains detected by high-resolution array in routine diagnostics. Eur J Hum Genet 20: 161-165 (2012).

Kang S-HL, Shaw C, Ou Z, Eng PA, Cooper ML, et al: Insertional translocation detected using FISH confirmation of array-comparative genomic hybridization (aCGH) results. Am J Med Genet A 152A:1111-1126 (2010).
Le Fevre AK, Taylor S, Malek NH, Horn D, Carr $\mathrm{CW}$, et al: FOXP1 mutations cause intellectual disability and a recognizable phenotype. Am J Med Genet A 161A:3166-3175 (2013).

Miller DT, Adam MP, Aradhya S, Biesecker LG, Brothman AR, et al: Consensus statement: chromosomal microarray is a first-tier clinical diagnostic test for individuals with developmental disabilities or congenital anomalies. Am J Hum Genet 86:749-764 (2010).

Nobukuni Y, Watanabe A, Takeda K, Skarka H, Tachibana M: Analyses of loss-of-function mutations of the MITF gene suggest that haploinsufficiency is a cause of Waardenburg syndrome type $2 \mathrm{~A}$. Am J Hum Genet 59:7683 (1996).

\footnotetext{
Schwaibold EMC, Zoll B, Burfeind P, Hobbiebrunken E, Wilken B, et al: A 3p interstitial deletion in two monozygotic twin brothers and an 18-year-old man: further characterization and review. Am J Med Genet A 161A:2634-2640 (2013).

- Țuțulan-Cunită AC, Papuc SM, Arghir A, Rötzer KM, Deshpande C, et al: $3 p$ interstitial deletion: novel case report and review. J Child Neurol 27:1062-1066 (2012).

- Van Hemel JO, Eussen HJ: Interchromosomal insertions. Identification of five cases and a review. Hum Genet 107:415-432 (2000).

Youings S, Ellis K, Ennis S, Barber J, Jacobs P: A study of reciprocal translocations and inversions detected by light microscopy with special reference to origin, segregation, and recurrent abnormalities. Am J Med Genet A 126A:46-60 (2004).
} 